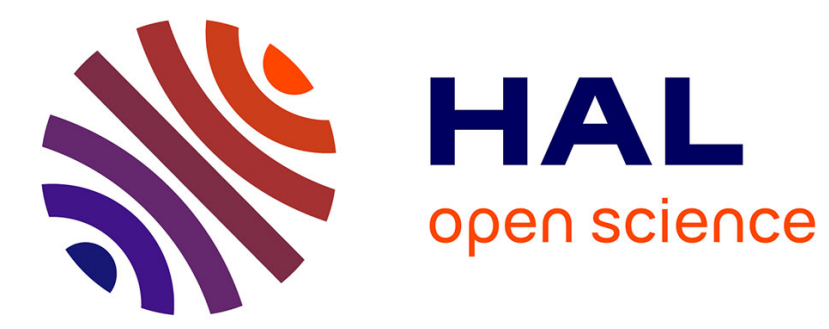

\title{
Angle Sensor Based on Chipless RFID Tag
}

Nicolas Barbot, Olivier Rance, Etienne Perret

\section{To cite this version:}

Nicolas Barbot, Olivier Rance, Etienne Perret. Angle Sensor Based on Chipless RFID Tag. IEEE Antennas and Wireless Propagation Letters, inPress. hal-02377065

\section{HAL Id: hal-02377065 https://hal.science/hal-02377065}

Submitted on 22 Nov 2019

HAL is a multi-disciplinary open access archive for the deposit and dissemination of scientific research documents, whether they are published or not. The documents may come from teaching and research institutions in France or abroad, or from public or private research centers.
L'archive ouverte pluridisciplinaire HAL, est destinée au dépôt et à la diffusion de documents scientifiques de niveau recherche, publiés ou non, émanant des établissements d'enseignement et de recherche français ou étrangers, des laboratoires publics ou privés. 


\title{
Angle Sensor Based on Chipless RFID Tag
}

\author{
Nicolas Barbot, Member, IEEE, Olivier Rance, Member, IEEE, and Etienne Perret, Senior Member, IEEE
}

\begin{abstract}
This paper presents theoretical and practical implementation of the chipless RFID technology to measure the orientation of tags attached on a 2D surface. Analytical expressions are used to recover tag orientation from mono-static co-polarization measurements. Simulations are performed to validate the orientation estimation technique. Moreover, measurements in anechoic chamber and real environment are done to evaluate the performance of the proposed solution. Contrary to already implemented techniques, the one introduced in this paper is not sensitive to distance variations which is an advantage for practical applications. Our solution can estimate the orientation of a chipless tag with an error less than $5^{\circ}$ in a range of $90^{\circ}$.
\end{abstract}

Index Terms-Chipless RFID, RCS, scatterer, angle sensor.

\section{INTRODUCTION}

Chipless RFID technology is a new paradigm to realize identification of items at a very low-cost compared to the classical RFID. However, the lack of a chip introduces new challenges in the tag design to store the data and to communicate this information to the reader. A lot of effort has already been made to increase coding capacity, to improve robustness of the reading and to reduce cost [1]. In [2], authors have shown that the price of a fully-printable chipless tag can be reduced to 0.4 euro cent making it a valid alternative or a complementary solution compared to classical RFID.

Like barcodes, chipless tags encode the information within the geometry of the tag so that memory is not required anymore. However to compete with barcodes, chipless RFID needs to propose new functionalities that are difficult or impossible to realize with the barcode technology. These functionalities can be added without altering the main identification function of the chipless tag. Indeed, tag ID can be extracted from the scatterers response, at the same time as other useful physical quantities that can give information about the tag vicinity. This additional information can be used to develop new kind of applications like sensing. Sensors based on chipless tags offer contactless, low-cost, and batteryless solutions which can be deployed in harsh environments. Many researchers have investigated the use of chipless tags to design new type of sensors for temperature [3]-[5], humidity [6], gas detection [7], level of fluids [8] and positioning [9].

Only few angle sensors using chipless tags are found in the literature. In [10], authors have designed a co-polarization chipless tag which exploits the polarization diversity to detect the tag orientation with a resolution of $20^{\circ}$. In [11], authors introduce a periodic structure printed on a grounded dielectric slab. Structure is rotated and authors show the backscattered field variations as a function of the orientation. Different orientations produce the same RCS value which limits the

The authors are with Univ. Grenoble Alpes, Grenoble INP, LCIS, F-26000 Valence, France.

M. Perret is also with Institut Universitaire de France, Paris, France. sensor range. In [12], same authors introduce a structure composed of 2 slightly different dipoles (without ground plane) with a relative orientation of $45^{\circ}$ to overcome the problem of null response. Orientation estimation is however limited to a range of $45^{\circ}$. Finally, in [13] authors consider the same finite periodic surface but with a scatterer composed of 4 different dipoles to determine the orientation. All these methods exploit the magnitude of the backscattered signal at the resonant frequency, and are thus sensitive to the distance between the antenna and the tag.

In this paper, new techniques are introduced to determine the orientation of a tag with an error less than $5^{\circ}$ in a range of $90^{\circ}$. Contrary to the previous studies the approach is based on an analytical model. The expressions are derived to be independent from the reading distance.

The paper is organized as follows, in Section II, the analytical expressions used to extract the tag orientation from red the measured S-parameters, are introduced. In Section III, two different chipless tags are presented and characterized for orientation sensing in anechoic chamber and real environment. Finally, Section IV concludes the paper.

\section{THEORY}

This section begins by determining the analytical relation between the measurand $\theta$ and the estimated quantity $\hat{\theta}$ obtained by measuring the backscattered S-parameters. It is shown that these relations can be greatly simplified by the use of thin dipoles as scatterers. Then, different methods are proposed to take into account the influence of distance variation.

\section{A. Rotation of a Scatterer}

Using the model described in [14], the S-parameter matrix $\mathbf{S}_{\mathrm{m}}$ measured at the VNA for a chipless tag can be expressed as:

$$
\mathbf{S}_{\mathbf{m}}=\mathbf{T} \cdot \mathbf{C} \cdot \mathbf{R}+\mathbf{T} \cdot \mathbf{S} \cdot \mathbf{R}
$$

where $\mathbf{T}$ and $\mathbf{R}$ represent the transmit path and received path respectively and are function of the distance $d$ between the tag and the antenna, $\mathbf{C}$ is the coupling between the emission and reception and $\mathbf{S}$ the polarization scattering matrix of the tag. Note that each term is a $2 \times 2$ matrix of complex and frequency dependent parameters in the vertical and horizontal plane. From (1), assuming $T_{v v} \gg T_{v h}$ and $R_{v v} \gg R_{v h}$ like in [14], the response in co-polarization of a chipless tag can be extracted:

$$
S_{11 m}=I_{v v}+R_{v v} \cdot S_{v v} \cdot T_{v v}
$$

where $I_{v v}=T_{v v} \cdot C_{v v} \cdot R_{v v}$ and can be estimated by measuring $S_{11 m}$ without the tag. Also, note that the quantity $S_{11 m}-I_{v v}$ is directly proportional to $S_{v v}$. Moreover, applying a rotation of $\theta$ to the scatterer (see Fig. 1) modifies the $\mathbf{S}$ matrix as [15]: 


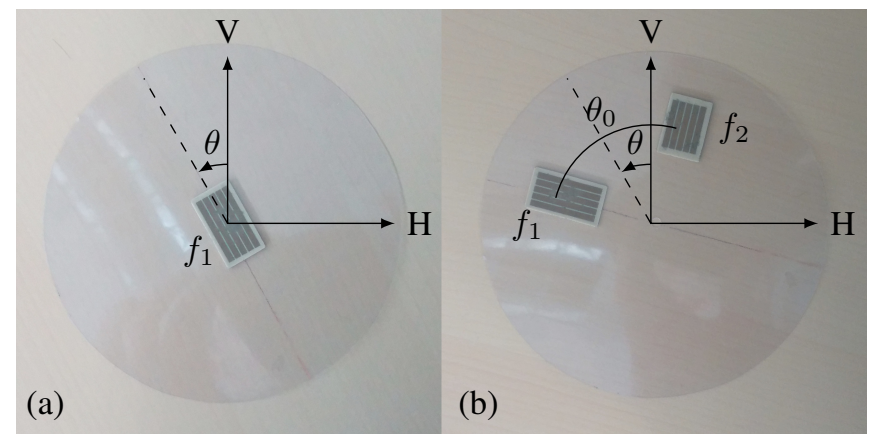

Fig. 1. Different tag configurations used: (a) with a single resonator, (b) with two resonators.

$$
\mathbf{S}(\theta)=\boldsymbol{\Omega}^{T} \cdot \mathbf{S} \cdot \boldsymbol{\Omega}
$$

where ${ }^{T}$ is the transpose operator and $\Omega$ is a rotation matrix defined by:

$$
\boldsymbol{\Omega}=\left[\begin{array}{cc}
\cos \theta & -\sin \theta \\
\sin \theta & \cos \theta
\end{array}\right]
$$

From (3), expression of $S_{v v}(\theta)$ can be written as:

$$
S_{v v}(\theta)=S_{v v} \cos ^{2} \theta+S_{v h} \sin 2 \theta+S_{h h} \sin ^{2} \theta
$$

Note that $S_{h h}(\theta)$ could also be expressed from (3). It is possible to solve (5) analytically to determine $\theta$. However, this estimation depends on $S_{v v}, S_{h h}$ and $S_{v h}$ which have to be known a priori, using at least three reference measurements, at the same distance $d$ than the orientation measurement $S_{v v}(\theta)$. If a single parameter is estimated at a different distance, (5) can not be used anymore.

An efficient way to greatly simplify (3) is to consider a thin dipole instead of a generic scatterer. For $\theta=0$, the scattering matrix $\mathbf{S}$ corresponding to the antenna mode of a dipole with a vertical orientation at the resonant frequency $f_{1}$ takes a simple form given by:

$$
\mathbf{S}^{f_{1}}(0)=\left[\begin{array}{cc}
S_{v v m a x}^{f_{1}} & 0 \\
0 & 0
\end{array}\right]
$$

where $S_{\text {vvmax }}^{f_{1}}$ depends on the distance between the tag and the antenna. When this thin dipole is rotated by an angle $\theta$ [see Fig. 1(a)], (3) simplifies into:

$$
\mathbf{S}^{f_{1}}(\theta)=S_{v v \max }^{f_{1}} \cdot\left[\begin{array}{cc}
\cos ^{2} \theta & \cos \theta \sin \theta \\
\cos \theta \sin \theta & \sin ^{2} \theta
\end{array}\right] .
$$

If we now consider a tag composed of two scatterers resonating at two different frequencies $f_{1}$ and $f_{2}$, separated by an angle $\theta_{0}$ [see Fig. 1(b)], and by supposing that the coupling between the two resonators can be neglected, (7) can still be expressed separately for the two resonators at their respective frequency simply by replacing $\theta$ by, $\theta-\theta_{0} / 2$ for $f_{1}$ and $\theta+\theta_{0} / 2$ for $f_{2}$. In all cases, $S_{v v \max }^{f_{i}}$ still depends on the distance between the tag and the antenna.

Fig. 2 presents the simulation of the scattered E-field in vertical co-polarization ( $\mathrm{VV}$ ) as a function of the frequency for a single resonator i.e., the tag configuration corresponding to Fig. 1(a). The resonance is observed at $3.5 \mathrm{GHz}$. As predicted by (7), the backscattered field is maximum at $\theta=0$ and null at $\theta=90^{\circ}$.

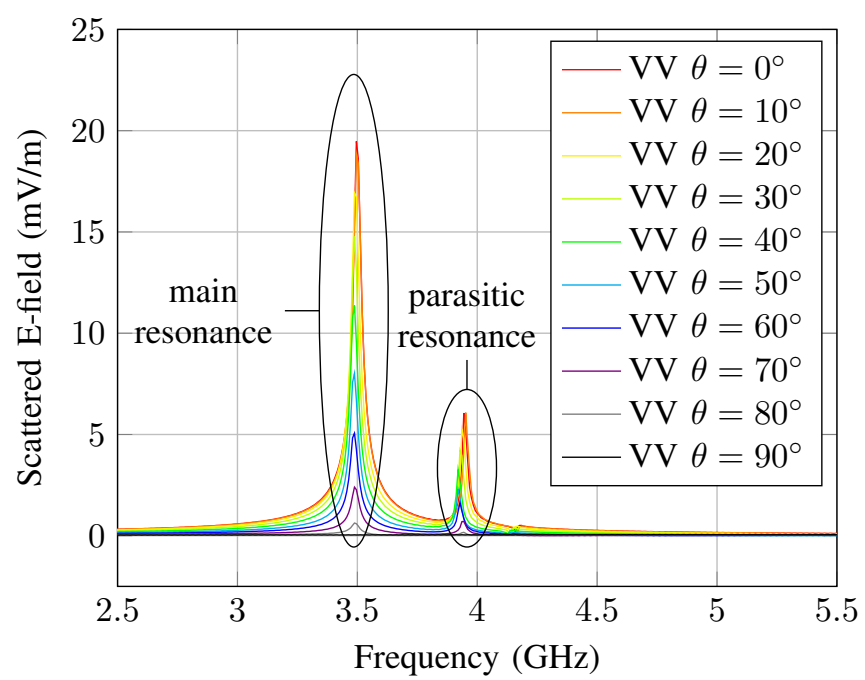

Fig. 2. Simulated scattered E-field in vertical co-polarization (VV) for the tag presented in Fig. 1(a) for different orientations using time gating.

\section{B. Orientation Determination in Co-polarization}

The relations developed in the previous section will be used to perform the extraction of the orientation. Three methods are presented, for which the objective is to determine an estimate of the orientation of the tag, noted $\hat{\theta}$, while minimizing the number of necessary measurements. A special attention is also given to the distance dependence.

The first one is a direct method which uses a single resonator [see Fig. 1(a)] and two measurements in vertical co-polarization. From (5) and (6), the vertical co-polarization component can be extracted as: $S_{v v}^{f_{1}}(\theta)=S_{v v m a x}^{f_{1}} \cos ^{2} \theta$. Taking the magnitude and isolating $\theta$ leads to:

$$
\hat{\theta}=\arccos \left(\sqrt{\frac{\left|S_{v v}^{f_{1}}(\theta)\right|}{\left|S_{v v \max }^{f_{1}}\right|}}\right)=\arccos \left(\sqrt{\frac{\mid S_{11 m}^{f_{1}}-I_{v v}^{f_{1} \mid}}{\left|S_{11 m}^{f_{1}}-I_{v v}^{f_{1}}\right|}}\right)
$$

where the second equality of (8) is obtained from (2) and is valid only if $T_{v v}^{f_{1}}(d) R_{v v}^{f_{1}}(d)$ are equal for both orientation measurement and reference measurement. This implies that the measurements have to be done at the same distance $d$.

However, it is possible to find expressions which do not depend on the distance. The second method also uses a single resonator [see Fig. 1(a)] and is based on the measurement of both vertical and horizontal component of the $\mathbf{S}$ matrix. From (7), $\theta$ can be obtained directly with the diagonal elements of the matrix $S_{v v \max }^{f_{1}} \cos ^{2} \theta$ and $S_{v v \max }^{f_{1}} \sin ^{2} \theta$, and the following formula can be derived:

$$
\hat{\theta}=\arctan \left(\sqrt{\frac{\left|S_{h h}^{f_{1}}(\theta)\right|}{\left|S_{v v}^{f_{1}}(\theta)\right|}}\right)=\arctan \left(\sqrt{\frac{\left|S_{22 m}^{f_{1}}-I_{h h}^{f_{1}}\right|}{\left|S_{11 m}^{f_{1}}-I_{v v}^{f_{1}}\right|}}\right)
$$

Assuming that antenna gain is the same at both access, the second equality remains valid for all distances as $T_{v v}^{f_{1}}(d) R_{v v}^{f_{1}}(d)$ and $T_{h h}^{f_{1}}(d) R_{h h}^{f_{1}}(d)$ are equal.

The third approach consists in using a tag with two resonators [see Fig. 1(b)], in this case, orientation determination can be done with a single measurement and a reference 
measurement both with a single-port antenna (by measuring $S_{v v}$ or $S_{h h}$ ). From (7), taking the magnitude and considering a relative angle between the resonators of $\theta_{0}=\pi / 2$, which involves that $\cos ^{2}\left(\theta+\theta_{0} / 2\right)=\sin ^{2}\left(\theta-\theta_{0} / 2\right)$, the tag orientation can simply be extracted and is equal to:

$$
\begin{aligned}
\hat{\theta} & =\arctan \left(\sqrt{\frac{\left|S_{v v m a x}^{f_{1}}\right| \cdot\left|S_{v v}^{f_{2}}\left(\theta+\theta_{0} / 2\right)\right|}{\left|S_{v v m a x}^{f_{2}}\right| \cdot\left|S_{v v}^{f_{1}}\left(\theta-\theta_{0} / 2\right)\right|}}\right)+\theta_{0} / 2 \\
& =\arctan \left(\sqrt{\frac{\left|S_{11 m}^{f_{1}}-I_{v v}^{f_{1}}\right| \cdot\left|S_{11 m}^{f_{2}}-I_{v v}^{f_{2}}\right|}{\left|S_{\text {max }_{2}}^{f_{2}}-I_{v v}^{f 2}\right| \cdot\left|S_{11 m}^{f_{1}}-I_{v v}^{f_{1}}\right|}}\right)+\theta_{0} / 2
\end{aligned}
$$

where $\left|S_{\text {vvmax }}^{f_{1}}\right|$ and $\left|S_{\text {vvmax }}^{f_{2}}\right|$ can be obtained with a single reference measurement at a known orientation. Moreover, since both numerator and denominator are scaled by $T_{v v}^{f_{1}}\left(d_{1}\right) T_{v v}^{f_{2}}\left(d_{2}\right),(11)$ holds even if orientation measurement and reference measurement are not done at the same distance from the antenna. Finally, for all methods, $\theta$ estimation belongs in a $90^{\circ}$ interval due to the positive sign argument of the inverse trigonometric functions.

\section{MeAsurement BenCH AND Results}

In the following, we describe the chipless tags and the measurement bench used to validate (9) and (11). Measurements are done in anechoic chamber and real environment.

\section{A. Tag Design and Characterization}

Two different chipless tags corresponding to the two proposed approaches have been realized for orientation determination (see Fig. 1). Note that the tag scatterers have been first introduced and optimized for identification purposes [14] Resonators include a ground plane to increase their selectivities. The first resonator has been designed to resonate at $3.5 \mathrm{GHz}$ and is based on five metallic stripes of dimensions $23 \times 2 \mathrm{~mm}$ separated by a gap of $0.5 \mathrm{~mm}$ which increase the RCS compared to the unitary dipole but introduce a parasitic resonance which is observed at $3.9 \mathrm{GHz}$ in Fig. 2 due to a different mode of resonance. The second resonator is based on the same structure except for the dipole's length which is $17.4 \mathrm{~mm}$ (which corresponds to a resonant frequency of $4.5 \mathrm{GHz}$ and a parasitic resonance of $5.15 \mathrm{GHz}$ ). Both tags use $0.8 \mathrm{~mm}$ thickness Rogers RO4003 as substrate $\left(\epsilon_{r}=3.55\right)$.

The measurement bench is presented in Fig. 3 and has been used to characterize the tags (see Fig. 1) in co-polarization (VV and $\mathrm{HH}$ ) at a distance of $10 \mathrm{~cm}$ (and $20 \mathrm{~cm}$ ) from the antenna. At these distances, the tag is in the radiating near field of the antenna [16]. Measurement has been done using the Vector Network Analyser (VNA) N5222A. Dualaccess dual-polarization Satimo QH2000 antenna has been connected to the VNA ports 1 and 2 in the horizontal and vertical polarization respectively. For comparison purposes, full wave simulations using CST Microwaves with transient solver have been performed. Note that time gating has been used to remove the structural mode of the simulations and measurement results [17].

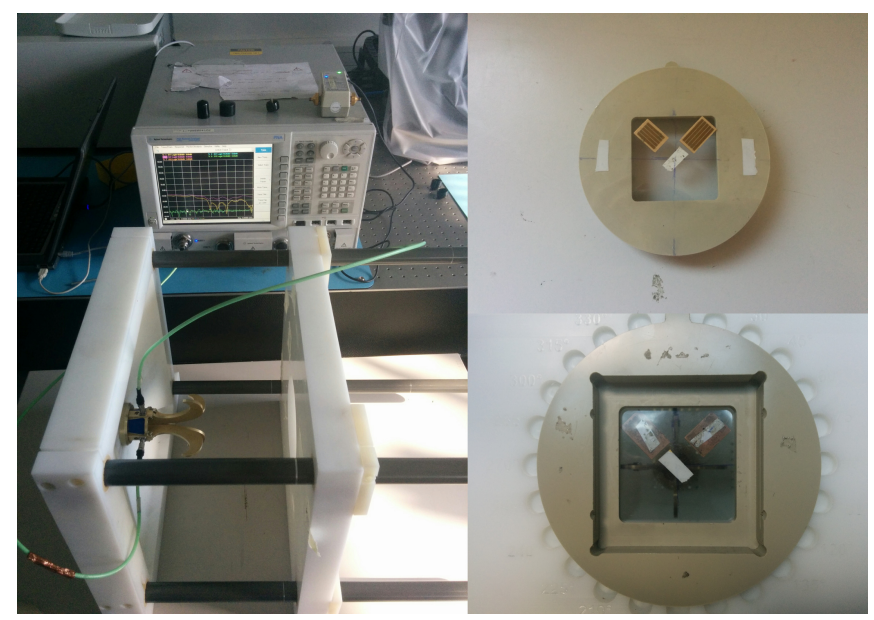

Fig. 3. Measurement bench used for tag orientation determination. A monostatic configuration is used with a dual polarization.

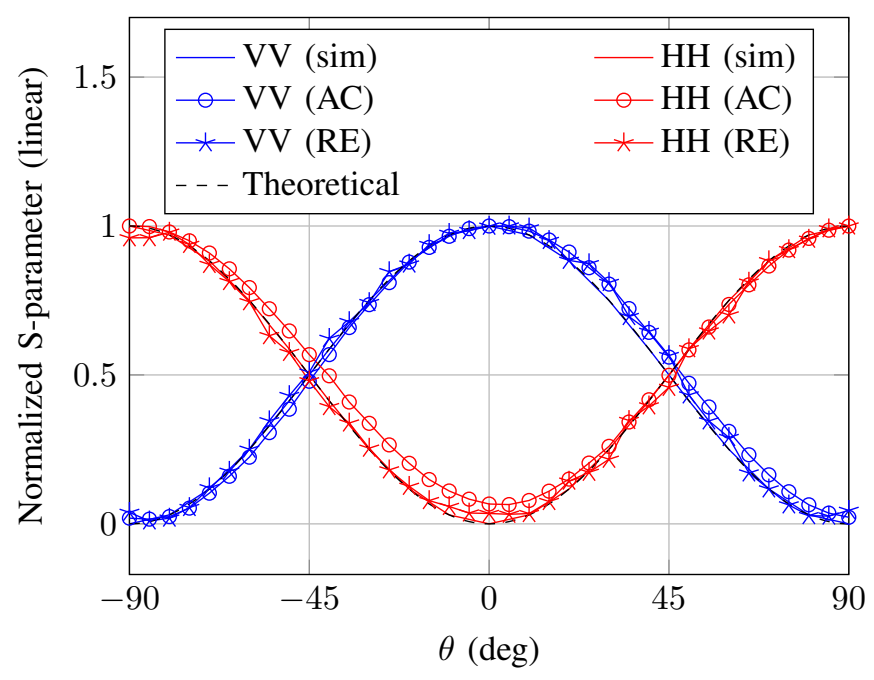

Fig. 4. Normalized scattered E-field peak apex value in co-polarization for the tag presented in Fig. 1(a) as a function of the orientation in simulation $(\mathrm{sim})$, measured in anechoic chamber (AC) and real environment (RE).

The impact of the tag orientation on the peak apex magnitude is represented in Fig. 4. The measured backscattered Efield in anechoic chamber and real environment is compared with both theory (7) and simulation. For comparison purposes, all results have been normalized to 1 . Simulations are in very good agreement with theory. Measurements present a slight offset compared to theory and simulation due to positioning errors and multi-path interference.

\section{B. Orientation Determination Using a Single Resonator}

As described in Section II-B, the tag orientation can be determined considering a single resonator using (8) and (9). Orientation estimation $\hat{\theta}$, obtained in real environment is plotted in Fig. 5 as a function of the real value of $\theta$. Orientation can be accurately derived in the interval $\left[0^{\circ} ; 90^{\circ}\right]$. In both cases, average value of the absolute orientation error is equal to respectively $2.85^{\circ}$ and $2.98^{\circ}$ for (8) and (9) over the complete interval. Differences between these two methods will be highlighted in Section III-C and III-D. 


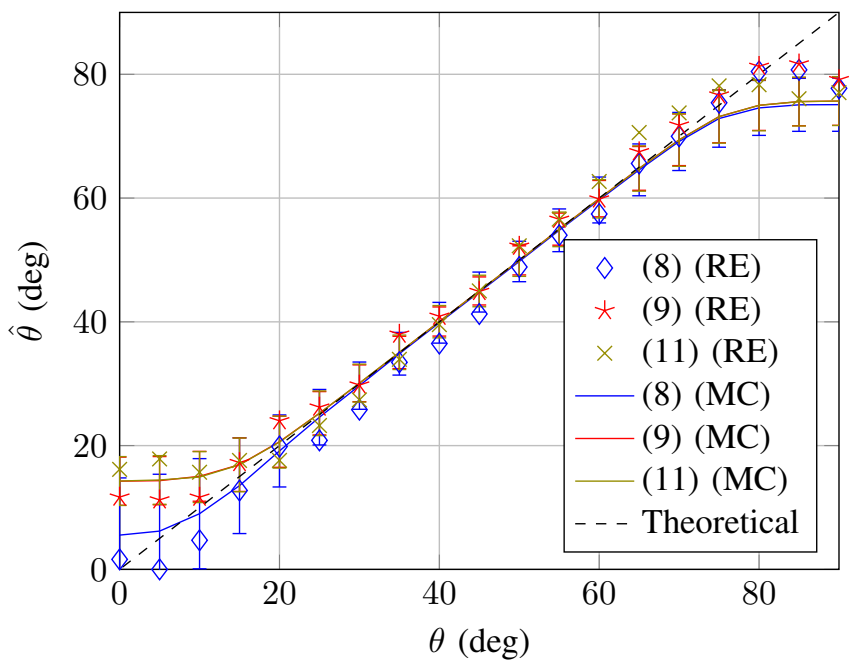

Fig. 5. Orientation estimation in co-polarization using (8), (9) and (11) obtained in real environment (RE) at $10 \mathrm{~cm}$, and using Monte Carlo (MC) method: average estimated value and standard deviation (vertical bars).

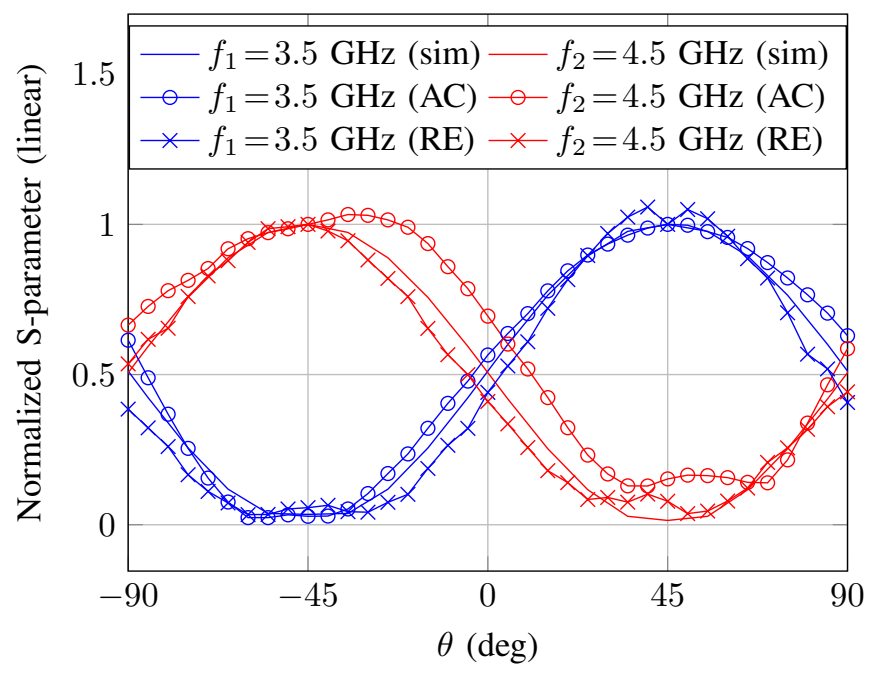

Fig. 6. Normalized scattered E-field of dual resonators presented in Fig. 1(b) with $\theta_{0}=90^{\circ}$ as a function of the orientation in simulation ( $\left.\operatorname{sim}\right)$, measured in anechoic chamber (AC) and real environment (RE).

\section{Orientation Determination Using Two Resonators}

As previously described, it is possible to determine the orientation using two resonators with a single port of the antenna using (11). Fig. 6 presents the back-scattered field in co-polarization as a function of $\theta$ for the tag presented in Fig. 1(b). For comparison purposes, all results have been normalized to 1 . We can see that the simulation and measurements are in good agreement. The maximum error between simulation and measurement occurs at $\theta= \pm 45^{\circ}$. Results of the tag orientation extraction using (11), between $-45^{\circ}$ and $45^{\circ}$, are plotted in Fig. 5. As for (8) and (9), we can observe that the accuracy of orientation estimation using (11) increases in the center of the interval. In average, the orientation error is equal to $4.6^{\circ}$ in real environment.
TABLE I

Average Estimation Error as a Function of the Distance (Real Environment) AND Noise (Monte CARlo Simulations)

\begin{tabular}{llllll}
\hline$d /$ SNR & $10 \mathrm{~cm}$ & $20 \mathrm{~cm}$ & $35 \mathrm{~dB}$ & $25 \mathrm{~dB}$ & $15 \mathrm{~dB}$ \\
\hline Equation (8) & $2.85^{\circ}$ & $23.6^{\circ}$ & $24.0^{\circ}$ & $2.87^{\circ}$ & $57.4^{\circ}$ \\
Equation (9) & $2.98^{\circ}$ & $4.70^{\circ}$ & $1.29^{\circ}$ & $3.32^{\circ}$ & $8.67^{\circ}$ \\
Equation (11) & $4.64^{\circ}$ & $5.05^{\circ}$ & $1.29^{\circ}$ & $3.33^{\circ}$ & $8.74^{\circ}$ \\
\hline
\end{tabular}

\section{Evaluation of the Distance and Noise Impact}

As we have seen previously, (9) and (11) permit to recover the orientation of a chipless tag independently from the distance. However, in real environment, signal to noise ratio decreases when distance increases. This section estimates the performance of the sensor as a function of the distance and noise. Two different distances have been chosen: $10 \mathrm{~cm}$ (as previously presented) and $20 \mathrm{~cm}$, both using the same measurement bench. As previously, the reference measurement has been chosen at $10 \mathrm{~cm}$ for both distances. Results obtained in real environment are presented in the two first columns of Table I where the average error is estimated for all configurations and for the two distances. We can observe that with (8), accuracy of the estimation depends on the distance i.e., the error becomes important if reference measurement and orientation measurement are not realized at the same distance. On the other side, with (9) and (11), the error is relatively independent of the distance (the slight increase is due to a lower signal to noise ratio). Since maximal errors appear at $0^{\circ}$ and $90^{\circ}$ (see Fig. 5), performance can be increased by considering a reduced angular range.

Concerning the noise effect, Monte Carlo simulations, presented in Fig. 5 and Table I, have been done to estimate the orientation error against additive white gaussian noise. For Fig. 5, SNR has been set to $25 \mathrm{~dB}$ which is consistent with the SNR level seen by the antenna when the tag is placed at 10 $\mathrm{cm}$. Average value (solid line) and standard deviation (vertical bars) of the error show that all the methods are affected by a bias and a higher standard deviation at $0^{\circ}$ and $90^{\circ}$. From Table I, note that (9) and (11) are more accurate when SNR is high and are characterized by same average error. Thus the proposed methods can estimate the orientation of a tag in real environment with a good accuracy, are not sensitive to distance variation, and are robust against noise.

\section{CONCLUSION}

In this paper, three different approaches have been presented to determine the tag orientation in co-polarization. Analytical formulas have been derived in all cases. Simulations and measurements have been performed to evaluate the performance of the proposed solutions. We have shown that our solution can estimate the orientation of the chipless tag with an error less than $5^{\circ}$. Moreover, two of the three methods presented in the paper are not sensitive to distance variation between the tag and the antenna. Finally, the same resonators can be used for reading the tag ID and for measuring the orientation so that tag's coding capacity is not reduced. Thus identification and sensing can be combined to address new kind of applications. 


\section{ACKNOWLEDGMENTS}

This research was funded by the European Research Council (ERC) under the European Union's Horizon 2020 research and innovation program (grant $\mathrm{N}^{\circ} 772539$ ).

\section{REFERENCES}

[1] A. Vena, E. Perret, and S. Tedjini, Chipless RFID based on RF encoding particle: realization, coding and reading system. Elsevier, 2016.

[2] A. Vena, E. Perret, S. Tedjini, G. E. P. Tourtollet, A. Delattre, F. Garet, and Y. Boutant, "Design of chipless RFID tags printed on paper by flexography," IEEE Trans. Antennas Propag., vol. 61, no. 12, pp. 58685877, 2013.

[3] D. Girbau, Á. Ramos, A. Lazaro, S. Rima, and R. Villarino, "Passive wireless temperature sensor based on time-coded UWB chipless RFID tags," IEEE Trans. Microw. Theory Techn., vol. 60, no. 11, pp. 36233632, 2012.

[4] B. Kubina, M. Schusler, C. Mandel, A. Mehmood, and R. Jakoby, "Wireless high-temperature sensing with a chipless tag based on a dielectric resonator antenna," in SENSORS, 2013 IEEE, Nov. 2013, pp. $1-4$.

[5] E. M. Amin and N. Karmakar, "Development of a chipless RFID temperature sensor using cascaded spiral resonators," in SENSORS, 2011 IEEE, Oct. 2011, pp. 554-557.

[6] Y. Feng, L. Xie, Q. Chen, and L.-R. Zheng, "Low-cost printed chipless RFID humidity sensor tag for intelligent packaging," IEEE Sensors J., vol. 15 , no. 6 , pp. 3201-3208, 2015.

[7] A. Vena, L. Sydänheimo, M. M. Tentzeris, and L. Ukkonen, "A fully inkjet-printed wireless and chipless sensor for $\mathrm{CO}_{2}$ and temperature detection," IEEE Sensors J., vol. 15, no. 1, pp. 89-99, 2015.

[8] A. Guillet, A. Vena, E. Perret, and S. Tedjini, "Design of a chipless RFID sensor for water level detection," in 201215 International Symposium on Antenna Technology and Applied Electromagnetics, June 2012, pp. $1-4$.
[9] N. Barbot and E. Perret, "A chipless RFID method of 2D localization based on phase acquisition," Journal of Sensors, vol. 2018, p. 6, 2018. [Online]. Available: https://www.hindawi.com/journals/js/2018/ $7484265 / \mathrm{cta} /$

[10] A. Vena, E. Perret, and S. Tedjini, "A compact chipless rfid tag using polarization diversity for encoding and sensing," in 2012 IEEE International Conference on RFID (RFID), April 2012, pp. 191-197.

[11] S. Genovesi, F. Costa, M. Borgese, A. Monorchio, and G. Manara, "Chipless RFID tag exploiting cross-polarization for angular rotation sensing," in 2016 IEEE International Conference on Wireless for Space and Extreme Environments (WiSEE), Sept. 2016, pp. 158-160.

[12] S. Genovesi, F. Costa, M. Borgese, F. A. Dicandia, A. Monorchio, and G. Manara, "Chipless RFID sensor for rotation monitoring," in 2017 IEEE International Conference on RFID Technology Application (RFIDTA), Sept. 2017, pp. 233-236.

[13] S. Genovesi, F. Costa, M. Borgese, F. A. Dicandia, and G. Manara, "Chipless radio frequency identification (RFID) sensor for angular rotation monitoring," Technologies, vol. 6, no. 3, 2018. [Online]. Available: http://www.mdpi.com/2227-7080/6/3/61

[14] A. Vena, E. Perret, and S. Tedjni, "A depolarizing chipless RFID tag for robust detection and its FCC compliant UWB reading system," IEEE Trans. Microw. Theory Techn., vol. 61, no. 8, pp. 2982-2994, 2013.

[15] E. F. Knott, J. F. Shaeffer, and M. T. Tuley, Radar cross section. SciTech Publishing, Inc., 2004

[16] A. Yaghjian, "An overview of near-field antenna measurements," IEEE Trans. Antennas Propag., vol. 34, no. 1, pp. 30-45, January 1986.

[17] A. Ramos, E. Perret, O. Rance, S. Tedjini, A. Lázaro, and D. Girbau, "Temporal separation detection for chipless depolarizing frequencycoded RFID," IEEE Trans. Microw. Theory Techn., vol. 64, no. 7, pp. 2326-2337, July 2016. 